\title{
Ecchymosis on the Plantar Side of the Foot
}

Cenker Eken $^{1}$ and Mustafa Serinken ${ }^{2 *}$

${ }^{1}$ Akdeniz University, Medical Faculty, Department of Emergency Medicine, Turkey

${ }^{2}$ Pamukkale University, Medical Faculty, Department of Emergency Medicine, Turkey

"Corresponding author: Mustafa Serinken, MD, Professor of Emergency Medicine, Pamukkale University Hospital, Department of Emergency Medicine, Denizli, Turkey, Tel: +90 25829660 00; E-mail: aserinken@hotmail.com

Received date: September 11, 2017; Accepted date: September 18, 2017; Published date: September 20, 2017

Copyright: (C) 2017 Eken C, et al. This is an open-access article distributed under the terms of the Creative Commons Attribution License, which permits unrestricted use, distribution, and reproduction in any medium, provided the original author and source are credited.

\begin{abstract}
Although the incidence of Lisfranc injuries is not high, they are related to long-term disability of the foot such as malalignment, functional deficits and weight bearing difficulties. Delayed treatment or missed diagnosis of Lisfranc injuries can result in significant complications. Lisfranc injuries result from both indirect and direct trauma. A 43 years old man came into the emergency with pain in his right foot after a direct trauma to the anterior part of his foot while playing soccer.Introduction

Lisfranc injuries are relatively uncommon, representing approximately $0.2 \%$ of all fractures. Although the incidence of Lisfranc injuries is not high, they are related to long-term disability of the foot such as malalignment, functional deficits and weight bearing difficulties [1]. Delayed treatment or missed diagnosis of Lisfranc injuries can result in significant complications.
\end{abstract}

\section{Case Report}

A 43 years old man came into the emergency with pain in his right foot after a direct trauma to the anterior part of his foot while playing soccer. He had pain over the dorsal side of the midfoot when palpated and an ecchymosis on the plantar arch of right foot (Figure 1).

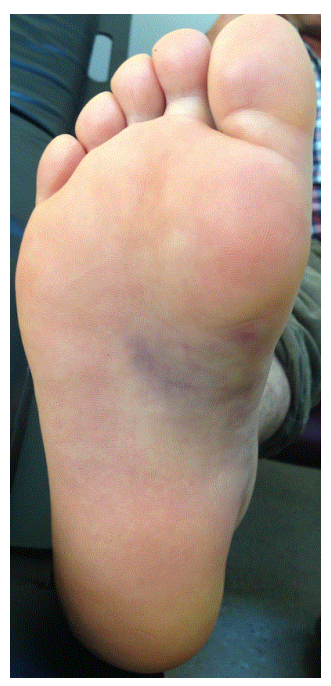

Figure 1: Ecchymosis on the plantar arch of the right foot.

There was no remarkable abnormality in the remaining physical examination. The plain radiograph is not remarkable but a possible fracture at the basis of first metatarsal bone (Figure 2).

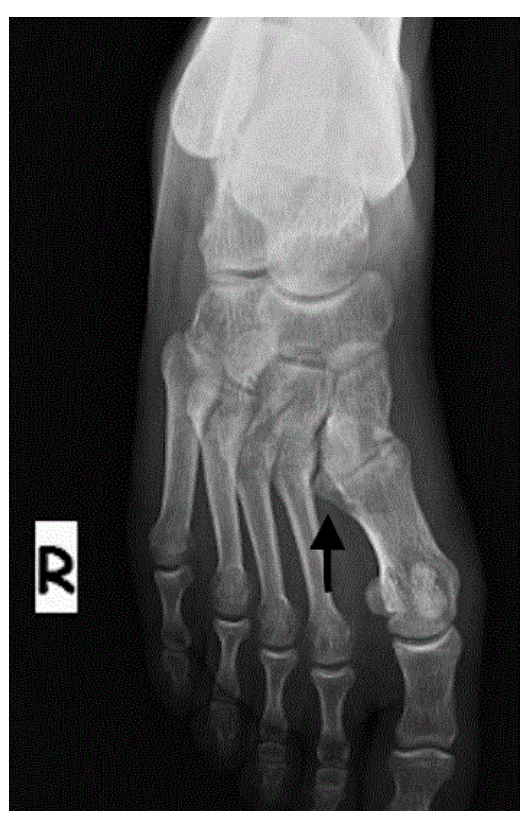

Figure 2: X-ray indicating a possible fracture on the basis of 1st metatarsal bone.

Computerized tomography revealed a separated fracture of the first metatarsal bone including tarsometatarsal joint (Figure 3). 
Page 2 of 2

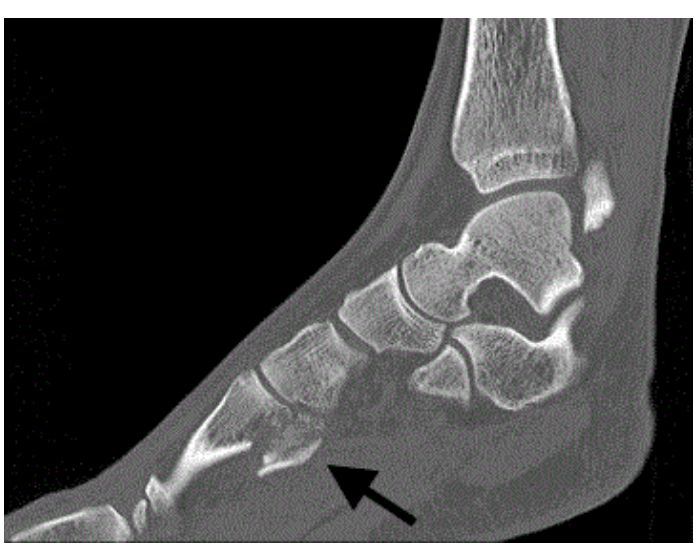

Figure 3: Computerized tomography displaying a separated fracture of 1st metatarsal bone involving the tarsometatarsal joint.

\section{Discussion}

Lisfranc joint represent the midfoot, which consists of tarsometatarsal joint. Lisfranc injury represent any injury, fracture or dislocation, to any of the structures of tarsometatarsal complex - the metatarsals, the tarsometatarsal joint, cuneiforms, cuboid and navicular [2]. Although the incidence of Lisfranc injuries is not high, they are related to long-term disability of the foot such as malalignment, functional deficits and weight bearing difficulties. Lisfranc joint allows the transition of force and weight from midfoot to forefoot which is crucial for gait. There are two common indirect mechanisms of Lisfranc injury: forced external rotation, or twisting of a pronated foot and axial loading of the foot in a fixed equinus position $[1,3]$.
Diagnosis is achieved by physical examination and radiology. Plantar arch ecchymosis in PE is pathognomic. Nevertheless, any malalignment between tarsal and metatarsal bones and a widening over $2.7 \mathrm{~mm}$ between the first and second basis of metatarsal bones may indicate an injury of Lisfranc joint. X-ray may also reveal any fracture or an obvious dislocation of the joint. Computerized tomography is the gold standard for Lisfranc injuries which provided exhaustive information concerning the fractures and dislocation of the area also allowing a more concise treatment plan $[3,4]$. X-rays are not always diagnostic because of multiple overlapping bones in the foot, particularly on the lateral view [1].

\section{Conclusion}

Delayed treatment or missed diagnosis of Lisfranc injuries can result in significant complications (degenerative arthritis, residual pain, compartment syndrome, loss of the metatarsal arch and unequal metatarsal pressure). Lisfranc fractures and dislocations require orthopedic consultation as the treatment requires operative fixation.

\section{References}

1. Welck MJ, Zinchenko R, Rudge B (2015) Lisfranc injuries. Injury 46: 536-541.

2. Schofer JM, O'Brien S (2008) Images in Emergency Medicine : Lisfranc Fracture-Dislocation. West J Emerg Med 9: 56-57.

3. Lau S, Bozin M, Thillainadesan T (2016) Lisfranc fracture dislocation: a review of a commonly missed injury of the midfoot. Emerg Med J 34: 52-56.

4. Miyamoto W, Takao M, Innami K, Miki S, Matsushita T (2015) Ligament reconstruction with single bone tunnel technique for chronic symptomatic subtle injury of the Lisfranc joint in athlete. Arch Orthop Trauma Surg 135: 1063-1070. 